OPEN ACCESS

Edited by:

Reza Lashgari,

Institute for Research in Fundamental

Sciences, Iran

Reviewed by:

Soroush Arabshahi,

Columbia University, United States

Kamran Avanaki,

University of Illinois at Chicago,

United States

*Correspondence:

Bin Zhang

xld_Jane_Eyre@126.com

Shuixing Zhang

shui7515@126.com

tThese authors have contributed equally to this work

Specialty section: This article was submitted to

Pulmonary Medicine,

a section of the journal

Frontiers in Medicine

Received: 19 December 2020

Accepted: 04 February 2021

Published: 25 March 2021

Citation:

Chen Q, Chen L, Liu S, Chen L, Li M, Chen Z, You J, Zhang B and Zhang S (2021) Three-Dimensional CT for

Quantification of Longitudinal Lung and Pneumonia Variations in

COVID-19 Patients.

Front. Med. 8:643917.

doi: 10.3389/fmed.2021.643917

\section{Three-Dimensional CT for} Quantification of Longitudinal Lung
and Pneumonia Variations in
COVID-19 Patients

\author{
Qiuying Chen ${ }^{\dagger}$, Lv Chen ${ }^{\dagger}$, Shuyi Liu, Luyan Chen, Minmin Li, Zhuozhi Chen, Jingjing You, \\ Bin Zhang* and Shuixing Zhang*
}

Department of Radiology, The First Affiliated Hospital of Jinan University, Guangzhou, China

Objectives: Visual chest CT is subjective with interobserver variability. We aimed to quantify the dynamic changes of lung and pneumonia on three-dimensional CT (3D-CT) images in coronavirus disease 2019 (COVID-19) patients during hospitalization.

Methods: A total of 110 laboratory-confirmed COVID-19 patients who underwent chest CT from January 3 to February 29, 2020 were retrospectively reviewed. Pneumonia lesions were classified as four stages: early, progressive, peak, and absorption stages on chest CT. A computer-aided diagnostic (CAD) system calculated the total lung volume (TLV), the percentage of low attenuation areas (LAA\%), the volume of pneumonia, the volume of ground-glass opacities (GGO), the volume of consolidation plus the GGO/consolidation ratio. The CT score was visually assessed by radiologists. Comparisons of lung and pneumonia parameters among the four stages were performed by one-way ANOVA with post-hoc tests. The relationship between the CT score and the volume of pneumonia, and between LAA\% and the volume of pneumonia in four stages was assessed by Spearman's rank correlation analysis.

Results: A total of 534 chest CT scans were performed with a median interval of 4 days. TLV, LAA\%, and the GGO/consolidation ratio were significantly decreased, while the volume of pneumonia, GGO, and consolidation were significantly increased in the progressive and peak stages (for all, $P<0.05$ ). The CT score was significantly correlated with the pneumonia volume in the four stages $(r=0.731,0.761,0.715$, and 0.669 , respectively, $P<0.001$ ).

Conclusion: 3D-CT could be used as a useful quantification method in monitoring the dynamic changes of COVID-19 pneumonia.

Keywords: coronavirus disease 2019, computed tomography, CT score, CAD system, low attenuation areas

\section{INTRODUCTION}

Rapid and accurate diagnosis is urgently needed for the coronavirus disease 2019 (COVID-19) pandemic. In addition to real-time fluorescence polymerase chain reaction (RT-PCR), chest CT has been extensively used as a convenient and highly sensitive tool for screening, diagnosis, and follow-up of COVID-19 since its outbreak (1). Bilateral peripheral ground-glass opacities (GGO) 
with or without consolidation are the most common lung appearance of COVID-19 (2). Some recent studies have depicted typical CT manifestations of COVID-19 pneumonia during disease course into the early, progressive, peak, and absorption stages (3-6). Semiquantitative CT score may reflect the severity of the disease, which is a simple marker in daily practice (7). However, radiologist-interpreted chest $\mathrm{CT}$ is limited by larger interobserver variability, time-consuming, and inefficient.

The serial lung changes in addition to pneumonia lesions were not taken into account in the follow-up plan of COVID-19 patients. The percentage of low-attenuation lung tissues can be used to describe the severities of the lung disease; low attenuation areas (LAAs) represent individual areas of emphysematous destruction (8). Previous studies failed to consider the coexisting chronic pulmonary abnormalities in COVID-19 patients, for instance, emphysema and interstitial lung diseases (7). Colombi et al. found that well-aerated lung volume on initial CT may indicate the severity of disease and was a predictor of intensive care unit (ICU) admission or death in patients with COVID-19 pneumonia (9). To date, no lung functional data are available for COVID-19 patients during hospitalization except for Mo et al. who found the impairment of lung volume in COVID-19 patients prior to discharge, especially in severe cases (10). Previous studies have shown that some recovered patients with other coronavirus pneumonias [e.g., severe acute respiratory syndrome (SARS) and Middle East respiratory syndrome (MERS)] may be left with persistently damaged lungs, which could last for months or even years (11-14). It would be of clinical importance to monitor the dynamic change of lung in COVID-19 patients after treatment and might determine the necessity for subsequent pulmonary function test and pulmonary rehabilitation.

In this study, we aimed to quantify the serial changes of lung and pneumonia on three-dimensional CT (3D-CT) in COVID19 patients using a computer-aided diagnostic (CAD) system, which provides the imaging means for medical professionals to rapidly and accurately evaluate disease severity and treatment response of COVID-19 patients. This may play an important role in disease monitoring, early intervention, and determination of the timing of admission.

\section{MATERIALS AND METHODS Study Population}

The retrospective study was approved by an ethics committee of our institution, and the informed consent was waived. A total of 185 patients were admitted to a designated hospital due to suspicion of COVID-19 between January 3 and February 29 , 2020. The inclusion criteria were as follows: (1) patients were positive for COVID-19 through RT-PCR for COVID-19 nucleic acid by throat swabs (at least two samples were taken, at least $24 \mathrm{~h}$ apart); (2) patients had at least one positive chest CT during hospitalization; and (3) patients had follow-up chest CT. The disease severity of COVID-19 was categorized into mild, moderate, severe, and critical illness on the basis of the

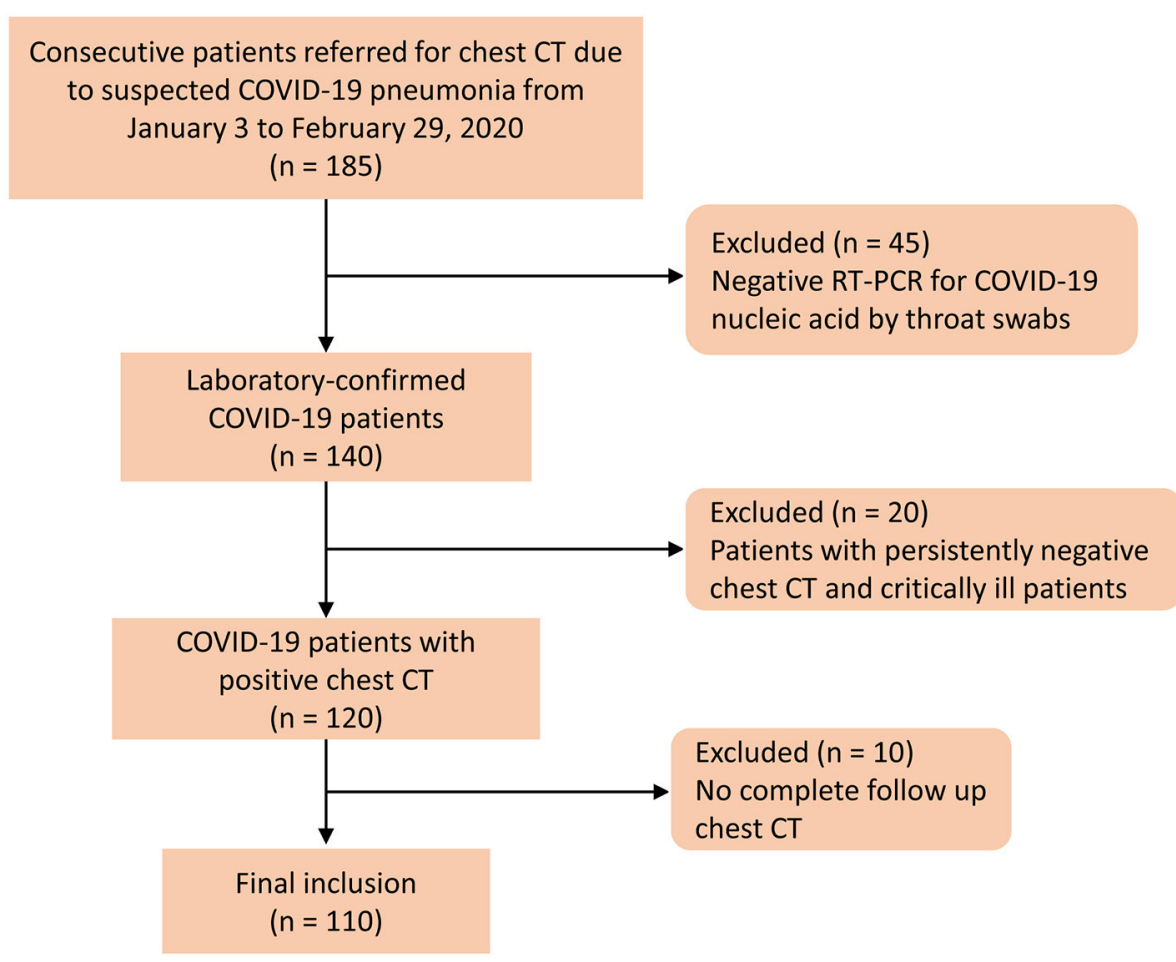

FIGURE 1 | Flow diagram of the patient selection process. Negative RT-PCR was defined as at least two repetitive negative RT-PCR test results, separated by at least 1 day. 

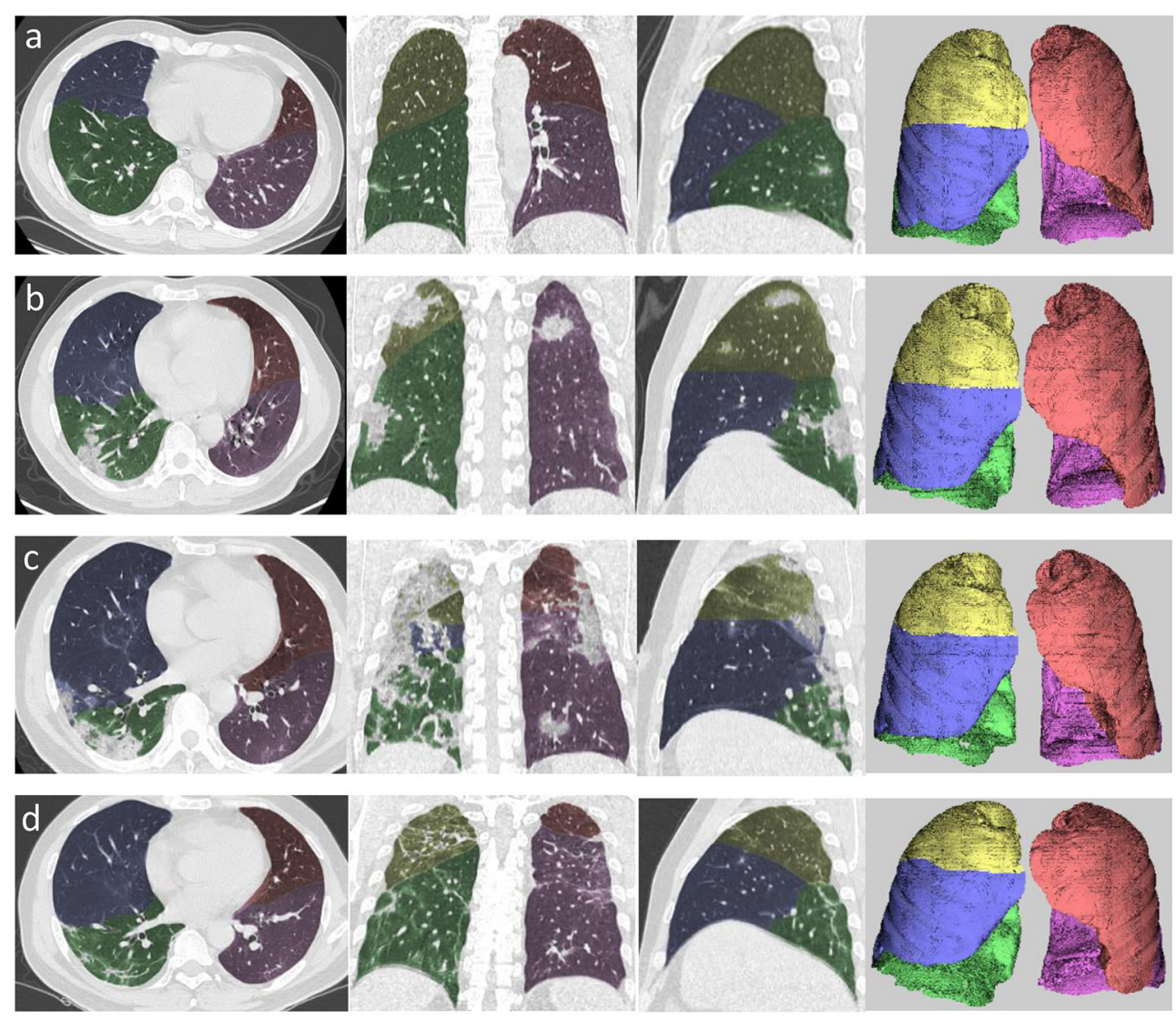

FIGURE 2 | Example of segmentation using automated computer-aided diagnosis in the four stages of COVID-19 pneumonia. (a) Axial, coronal, and sagittal multiplanar reconstruction views and volume rendering in the early stage. (b) Axial, coronal, and sagittal multiplanar reconstruction views and volume rendering (left to right) in the progressive stage. (c) Axial, coronal, and sagittal multiplanar reconstruction views and volume rendering (left to right) in the peak stage. (d) Axial, coronal, and sagittal multiplanar reconstruction views and volume rendering (left to right) in the absorption stage. Yellow area denotes right upper lobe, blue area denotes right middle lobe, green area denotes right lower lobe, orange area denotes left upper lobe, and pink area denotes left lower lobe.

newest COVID-19 guidelines released by the National Health Commission of China (15). Patients had persistently negative chest CT, and critically ill patients who underwent X-rays were excluded. Figure 1 shows the patients' enrollment flowchart. Finally, 110 COVID-19 patients were included for analysis.

\section{CT Examinations}

All patients underwent unenhanced chest CT scans by a Siemens Emotion 16 scanner (Siemens Healthineers; Erlangen, Germany), a CT 64 scanner (GE Medical System), or an ICT 128 scanner (Philips Healthcare, Netherlands). No contrast agent was administered. CT acquisition of the GE 64 scanner was executed as follows: tube voltage, $120 \mathrm{kV}$; tube current, $260 \mathrm{mAs}$; pitch, 0.984; and slice thickness reconstructions of $0.625 \mathrm{~mm}$. CT acquisition of the Siemens 16 scanner was executed as follows: tube voltage, $130 \mathrm{kV}$; automatic tube current; pitch, 1.5; and slice thickness reconstructions of 1.0 or $0.6 \mathrm{~mm}$. CT acquisition of the ICT 128 scanner was executed as follows: tube voltage, 120 $\mathrm{kV}$; automatic tube current; pitch, 0.7 ; collimation, $0.625 \mathrm{~mm}$ and slice thickness reconstructions of 1.0 or $0.67 \mathrm{~mm}$.

\section{Quantitative CT Analysis}

According to a previous study (3), early, progressive, peak, and absorption stages on chest CT were defined as $0-4,5-8,9-13$, and $\geq 14$ days after the onset of the initial symptoms, respectively. A CT scoring system was used to assess the involvement area/degree of pneumonia for each lung lobe: 0 for $0 \% ; 1$ for $1-25 \%$; 2 for $26-50 \%$; 3 for $51-75 \%$; and 4 for $76-100 \%$ (2). A CT score (range, 0-20) was assigned by summarizing the total scores for the five lobes. All the chest CT images were reviewed independently by two radiologists (with more than 10 years of experience), who were blinded to clinical and laboratory results. Any discrepancy was resolved by a consensus viewing.

The chest CT images were transferred to a workstation (Synapse Image Intelligence $^{\mathrm{TM}}$ Vincent version 4.4; Fujifilm Medical Systems, Tokyo, Japan). This workstation implemented a lobar CAD system that was showed to accurately measure lobar volumes (16). This system automatically extracted both lungs, recognized lobar bronchi, and determined the locations of fissures (Figure 2). Subsequently, the CAD system semiautomatically extracted pneumonia regions after simply dragging both ends of the infected regions. Two cardiothoracic radiologists 

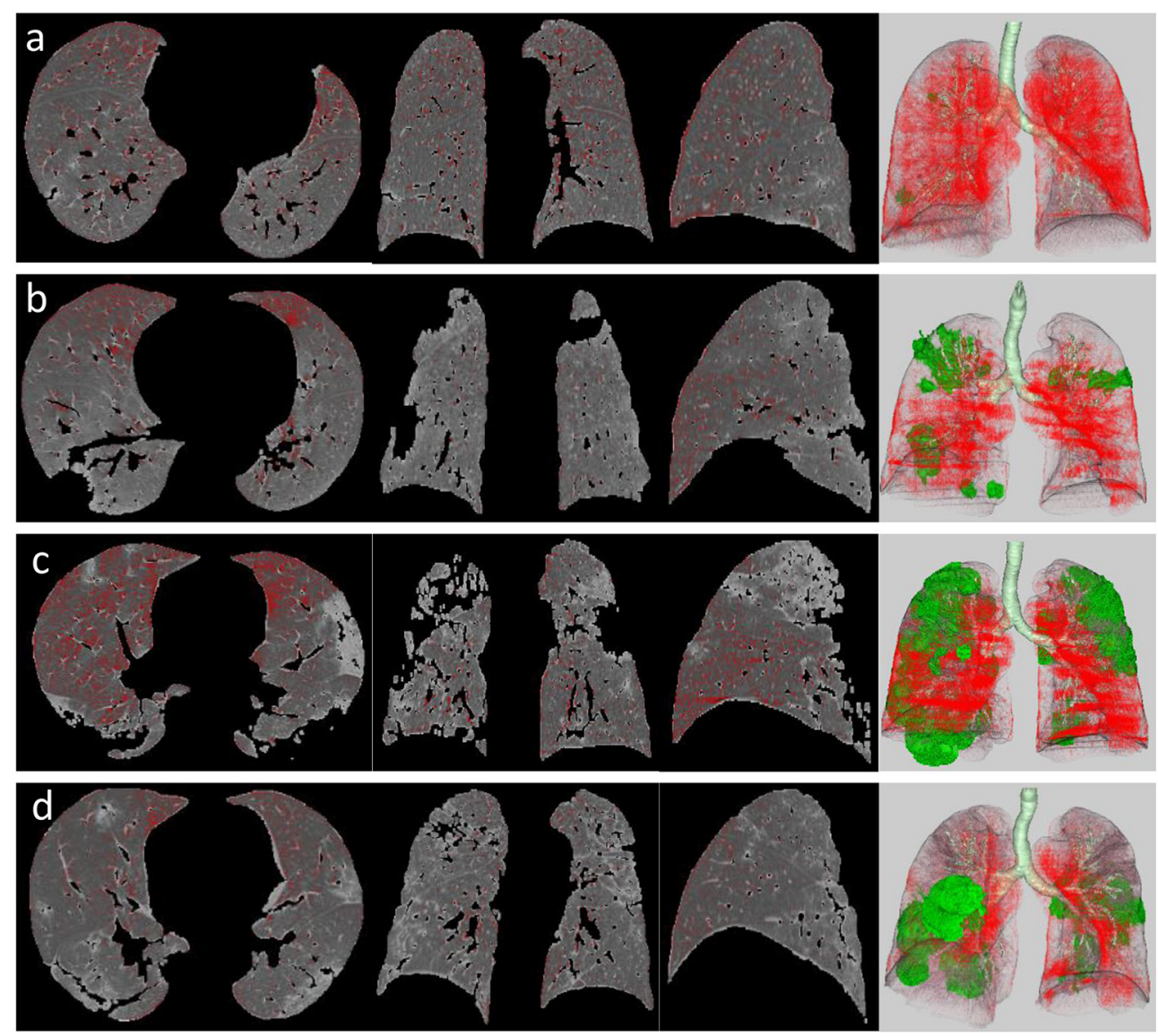

FIGURE 3 | LAA views of the four stages in a patient with COVID-19 pneumonia, which was defined as the lung field area with attenuation values <-950 HU of threshold (red areas). It shows axial, coronal, and sagittal two-dimensional (2D) displays and three-dimensional (3D) image (left to right) of LAA distribution in the early stage (a), progressive stage (b), peak stage (c), and absorption stage (d). Green area on 3D image represents pneumonia lesions.

checked the lesion segmentation by $\mathrm{CAD}$ and made manual corrections by delineating fissures and contour of infected regions when the CAD system failed to properly identify these.

A quantitative analysis procedure was performed based on the segmentation results. The lung field area with attenuation values $<-950$ Hounsfield Unit (HU) of thresholds was considered as LAAs (Figure 3). The proportion of LAAs (LAA\%) for total lung volume was calculated automatically. By thresholding on CT values in the pneumonia lesions, GGO and consolidation were identified with the value range of -800 to $-300 \mathrm{HU}$ and -300 to $50 \mathrm{HU}$, respectively (17) (Figure 4). The volume and percentage of GGO and consolidation were calculated, accordingly. The ratio of GGO to consolidation was also computed.

\section{Statistical Analysis}

Total lung volume, LAA $\%$, the volume of pneumonia, the volume of consolidation, the percentage of GGO, the percentage of consolidation, and the GGO/consolidation ratio obtained at the four stages were compared using one-way ANOVA with posthoc LSD multiple comparison tests at a level of significance $P<$ 0.05 . Spearman's rank correlation analysis was used to assess the correlation between the CT score and the volume of pneumonia.
All statistical analyses were performed using the SPSS statistical software package (version 23, SPSS Inc., Chicago, IL, USA).

\section{RESULTS}

\section{Clinical Characteristics}

The mean age of the 110 patients was $45.2 \pm 12.8$ years (range, $14-80$ years) and $65(59.1 \%)$ were males. A total of 100 patients (90.9\%) had moderate illness, and 10 patients (9.1\%) had severe illness. A total of 534 chest CT scans were conducted with a median of 4 scans (interquartile range, 3-5) per patient. The total CT scans for the early, progressive, peak, and absorption stages were 91 (17.0\%), $188(35.2 \%), 39(7.3 \%)$, and $216(40.4 \%)$, respectively. A total of 62 (56.4\%), 89 (80.9\%), 18 (16.4\%), and $95(86.4 \%)$ patients experienced early, progressive, peak, and absorption stages, respectively.

\section{Comparison of Lung and Pneumonia Parameters Generated by the CAD System}

Table 1 shows the comparison of lung and pneumonia parameters among the four stages. As compared with the CT score of early stage, CT scores of the progressive stage (7.6 \pm 3.2 


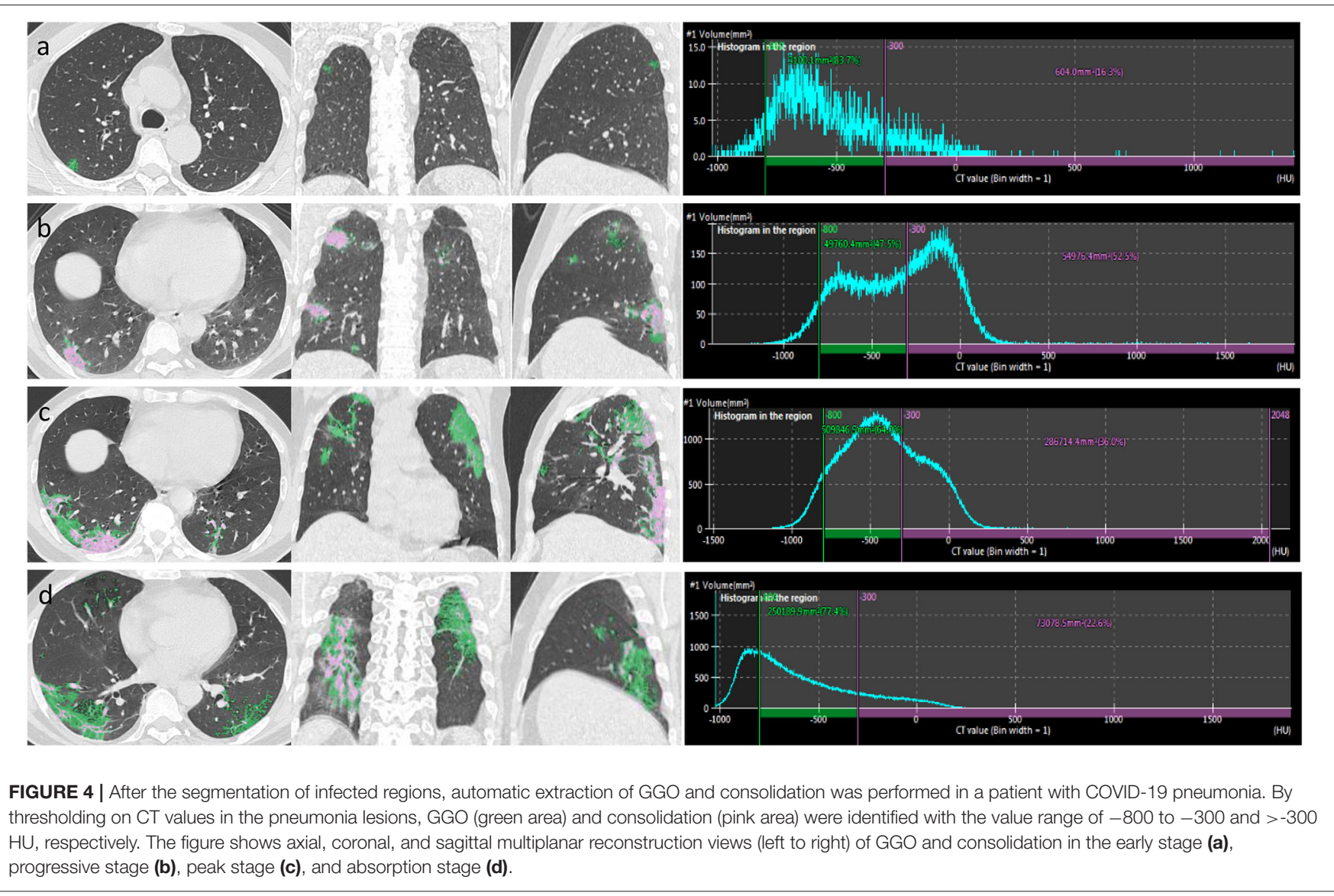

vs. $3.2 \pm 2.1, P<0.001)$ and the peak stage $(10.3 \pm 4.4$ vs. 3.2 $\pm 2.1, P<0.001)$ were significantly higher. Table 1 shows that the total lung volume significantly decreased in the progressive stage $(3.5 \mathrm{~L} \pm 1.1$ vs. $4.2 \mathrm{~L} \pm 1.2, P<0.001)$ and the peak stage $(3.1 \mathrm{~L} \pm 0.9$ vs. $4.2 \mathrm{~L} \pm 1.2, P<0.001)$ and then recovered in the absorption stage $(3.8 \mathrm{~L} \pm 1.1$ vs. $4.2 \mathrm{~L} \pm 1.2, P=0.008)$. The bilateral lower lobes were the most infected. The volume of pneumonia was significantly increased in the progressive stage $\left(431.5 \pm 409.3 \mathrm{~cm}^{3}\right.$ vs. $\left.73.8 \pm 96.0 \mathrm{~cm}^{3}, P<0.001\right)$ and the peak stage ( $568.1 \pm 470.6 \mathrm{~cm}^{3}$ vs. $\left.73.8 \pm 96.0 \mathrm{~cm}^{3}, P<0.001\right)$ and then decreased in the absorption stage $\left(291.0 \pm 404.6 \mathrm{~cm}^{3}\right.$ vs. $73.8 \pm$ $\left.96.0 \mathrm{~cm}^{3}, P<0.001\right)$. The mean time to peak of the pneumonia volume was $5.2 \pm 4.8$ days. In the early stage, 22 patients $(20 \%)$ had LAA $\%$ of $<5 \%, 78$ patients (70.9\%) had LAA\% of $5-24 \%$, and 10 patients (9.1\%) had LAA\% of $25-49 \%$. LAA $\%$ decreased in the progressive stage $(10.1 \pm 6.6 \%$ vs. $13.7 \pm 8.0 \%, P<0.001)$, the peak stage $(10.3 \pm 5.7 \%$ vs. $13.7 \pm 8.0 \%, P<0.01)$, and the absorption stage $(9.8 \pm 6.1 \%$ vs. $13.7 \pm 8.0 \%, P<0.001)$. GGO volume increased in the progressive stage $\left(230.6 \mathrm{~cm}^{3} \pm 231.0 \mathrm{vs}\right.$ $\left.41.4 \mathrm{~cm}^{3} \pm 59.2, P<0.001\right)$, peak stage $\left(328.3 \mathrm{~cm}^{3} \pm 299.0\right.$ vs $\left.41.4 \mathrm{~cm}^{3} \pm 59.2, P<0.001\right)$, but decreased in the absorption stage $\left(155.4 \mathrm{~cm}^{3} \pm 204.7\right.$ vs $\left.41.4 \mathrm{~cm}^{3} \pm 59.2, P<0.001\right)$. Consolidation volume increased in the progressive stage $\left(125.6 \mathrm{~cm}^{3} \pm 122.1 \mathrm{vs}\right.$ $\left.21.6 \mathrm{~cm}^{3} \pm 30.9, P<0.001\right)$, peak stage $\left(157.7 \mathrm{~cm}^{3} \pm 124.4 \mathrm{vs}\right.$ $\left.21.6 \mathrm{~cm}^{3} \pm 30.9, P<0.001\right)$, but decreased in the absorption stage $\left(49.4 \mathrm{~cm}^{3} \pm 71.5\right.$ vs $\left.21.6 \mathrm{~cm}^{3} \pm 30.9, P<0.05\right)$. GGO: consolidation ratio was significantly reduced in the progressive stage $(3.0 \pm 4.0$ vs $31.2 \pm 160.5, P<0.01)$, peak stage $(2.6 \pm 2.7$ vs $31.2 \pm 160.5, P<0.05)$ but increased in the absorption stage $(9.5 \pm 36.2$ vs $31.2 \pm 160.5, P<0.05)$.

\section{Relationship Between CT Score and Volume of Pneumonia}

Figure 5 shows the association between the visual CT score and the volume of pneumonia measured by the CAD system in the four stages. The CT score was found to be significantly correlated with the volume of pneumonia in the early stage $(r=0.731,95 \%$ CI: $0.614-0.816, P<0.001)$, the progressive stage $(r=0.761,95 \%$ CI: $0.692-0.817, P<0.001)$, the peak stage $(r=0.715,95 \% \mathrm{CI}$ : $0.508-0.844, P<0.001)$, and the absorption stage $(r=0.669,95 \%$ CI: $0.585-0.738, P<0.001)$.

\section{DISCUSSION}

In this study, we quantified the dynamic changes in lung and pneumonia on 3D-CT in patients with COVID-19 during hospitalization. The results showed that the lung volume and LAA\% significantly decreased in the progressive, peak, and absorption stages. The volume of pneumonia lesions, GGO, and consolidation continually increased in the progressive and peak stages. The GGO/consolidation ratio was significantly reduced in the progressive and peak stages and then recovered a little in the 
TABLE 1 | Comparison of lung and pneumonia parameters generated by the CAD system among the four stages.



Values are expressed as mean (SD). " One-way ANOVA with post-hoc LSD multiple comparison tests was used to test the differences between data at early, progressive, peak, and absorption stages. The early stage was used as a reference. ${ }^{+} P<0.05,{ }^{++} P<0.01$, and ${ }^{+++} P<0.001$. LUL, left lower lobe; LUL, left upper lobe; $R L L$, right lower lobe; $R M L$, right middle lobe; RUL, right upper lobe; LAA\%, percentage of low attenuation areas; GGO, ground-glass opacities.

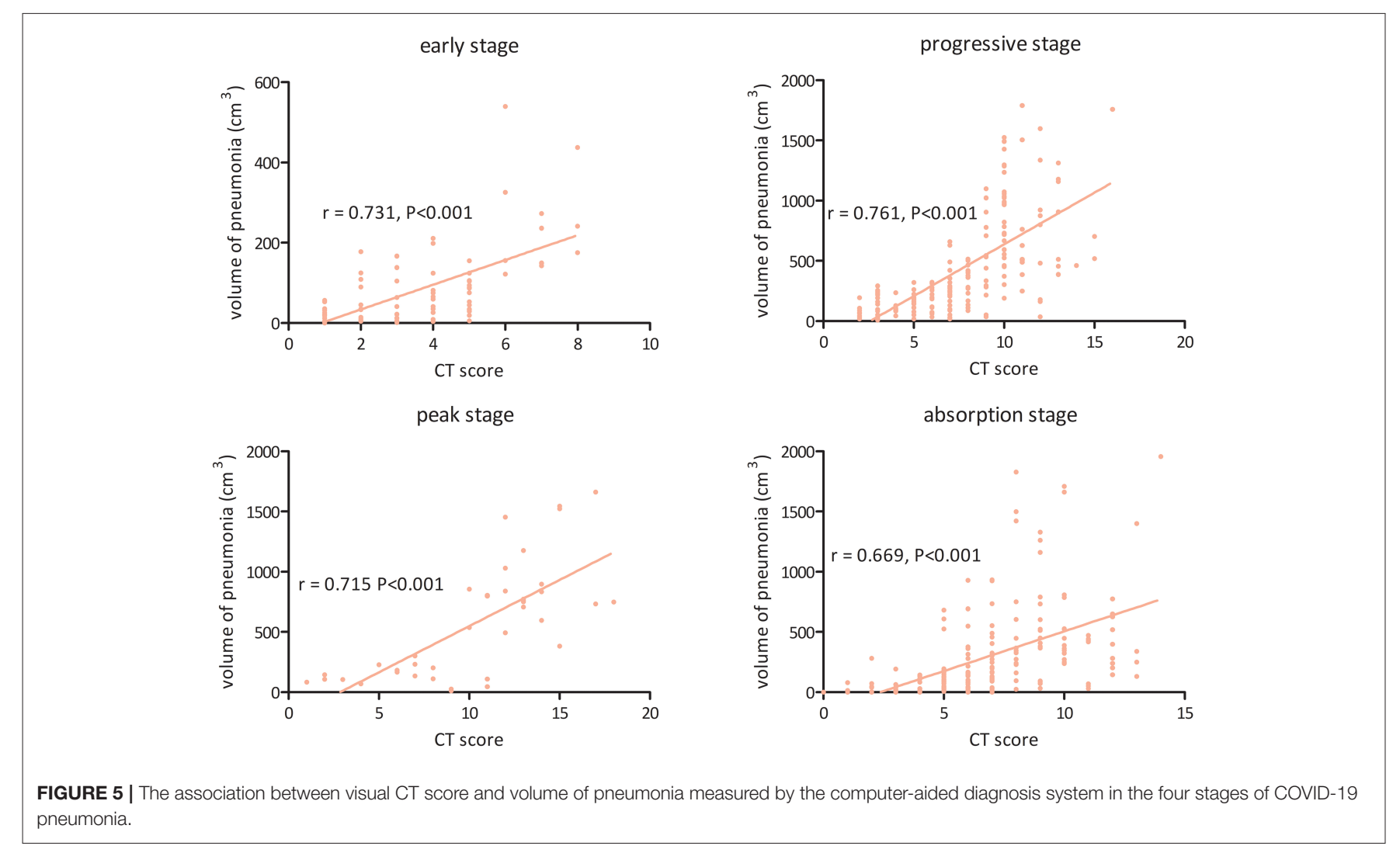


absorption stage. In addition, we found a moderate association between the visual CT score and the volume of pneumonia in the early, progressive, and absorption stages.

The lung is the most involved organ by COVID-19 (18), and typical pathological findings consist of diffuse alveolar epithelium destruction, alveolar septal fibrous proliferation, hyaline membrane formation, and capillary damage/bleeding (19). The pathological changes are the basis of typical CT findings of COVD-19 pneumonia. Previous studies observed the timedependent changes of COVID-19 pneumonia in the extent and severity of lesions $(3,20,21)$, which could be classified as four stages. In the early stage, bilateral GGO with subpleural distribution was the primary manifestation. Subsequently, the infection rapidly progressed to diffuse GGO with crazy-paving pattern, air bronchogram sign, and consolidation. After that, infections in some patients increased to peak involvement in regard to size, number, and density. After the infection was controlled by supportive treatment, the consolidation was absorbed gradually with fibrosis, but GGO appeared due to the absorption of consolidation. Our quantitative analyses perfectly reflected the time course of lung changes on chest CT. We observed a significantly increased volume of pneumonia lesions, GGO, and consolidation in the progressive stage, which slowly increased in the peak stage and significantly decreased in the absorption stage. The mean time to peak of the pneumonia volume was $5.2 \pm 4.8$ days. The GGO/consolidation ratio was persistently reduced in the progressive and peak stages and then recovered a little in the absorption stage. Timely monitoring the changes of total pneumonia, GGO, and consolidation would be beneficial for the evaluation of treatment response and adjustment of the clinical staging of COVID19 patients.

The semiquantitative CT score can assess the disease severity of COVID-19 according to the area or degree of lung involvement (3). This marker is simple and readily available in a clinical setting without any need of post-processing. However, it is somewhat subjective when the COVID-19 pneumonia lesions are multiple and irregular. CAD-based quantitative CT might outperform the traditional semiquantitative method based on the visual CT score. Despite the limitation of the CT score, we found a moderate correlation between the visual CT score and the volume of pneumonia measured by our CAD system, indicating that the CT score could be safely and effectively used in clinical practice. Our study showed that the CT scores of progressive and peak stages were significantly higher than those of the early and absorption stages, which may be correlated with elevated inflammation-related biomarkers (22). Yang et al. showed that the CT score was higher in severe cases as compared to mild cases (23). They found that using the CT score of larger than 19.5 could predict severe COVID-19, with high sensitivity (83\%) and specificity (94\%), which was consistent with the study of Liu et al. (24). Mahdjoub et al. found that the admission CT score was an independent predictor for a 5-day outcome (i.e., mechanical ventilation or death) of COVID-19 patients (25). Therefore, the CT score may help risk stratification and identify those patients at high risk of rapid progress who need timely treatment.
To the best of our knowledge, this study firstly demonstrated that the lung volume of COVID-19 significantly decreased in the progressive and peak stages and then slowly increased in the absorption stage on chest CT, which may suggest the impairment of pulmonary function in COVID-19 patients; but, in the future, a pulmonary function test (PFT) is needed to confirm. Previous studies showed that a significant correlation between lung volume and PFT results in interstitial lung diseases (26-28). Since the outbreak of this disease, its impact on lung function remains unknown. Previous studies have demonstrated that recovered patients with SARS-CoV and MERS-CoV may be left with persistently damaged lung function (11-14). Mo et al. revealed that, in COVID-19 survivors, the most common abnormality of lung function is the reduced diffusion capacity, followed by restrictive ventilatory defect, which is associated with the disease severity (10). A recent case report might suggest that older patients with COVID-19 were more likely to have residual radiological changes and impaired lung function after discharge (29). These preliminary findings might suggest that lung function monitoring and rehabilitation in patients who recovered from COVID-19 is necessary. Our study may provide a possibility to evaluate lung volume without additional health costs.

Our study also has some limitations. First, it is a retrospective study. Second, our selection criteria might have introduced selection bias since we excluded mild and critical illness. Third, the different CT scan parameters might have a potential impact on software-based quantification. Fourth, some patients could not hold well their breath, especially severe cases, which may pose an impact on the calculation. Fifth, the sample size is relatively small, specifically for the peak stage; more subjects are needed to better understand the infection's underlying mechanism and its spread pattern. Sixth, the impact of other patient demographics, such as smoking status and preconditioned respiratory disease wasn't identified, which may play as a confounder. Finally, the effect of other lung structures, such as airways, vessels, and fissures, wasn't observed for analysis.

In summary, quantitative $3 \mathrm{D}-\mathrm{CT}$ could be used as a useful complementary method to conventional CT in the follow-up of COVID-19 patients. Quantitative assessment of the dynamic changes in lung and pneumonia in patients with COVID-19 may be useful for routine patient management.

\section{DATA AVAILABILITY STATEMENT}

The original contributions generated in the study are included in the article/supplementary material, further inquiries can be directed to the corresponding authors.

\section{ETHICS STATEMENT}

The studies involving human participants were reviewed and approved by the Ethics Committee of The First Affiliated Hospital of Jinan University. Written informed consent for participation was not required for this study in accordance with the national legislation and the institutional requirements. 


\section{AUTHOR CONTRIBUTIONS}

QC and LC contributed to the conception and design of the study, the analysis and interpretation of data, and the work drafting. SL, LC, and ML participated in the data extraction and analysis. $\mathrm{ZC}$ and JY designed figures. BZ and SZ offered guidance in study design and revised the article critically for important intellectual content. All authors read the revision of the article as well as final approval of the version to be submitted.

\section{FUNDING}

This work was supported by a grant from the National Natural Science Foundation of China (Grant Numbers:

\section{REFERENCES}

1. Rubin GD, Ryerson CJ, Haramati LB, Sverzellati N, Kanne JP, Raoof S, et al. The role of chest imaging in patient management during the COVID-19 pandemic: a multinational consensus statement from the fleischner society. Radiology. (2020) 296:172-80. doi: 10.1148/radiol.2020201365

2. Chung M, Bernheim A, Mei X, Zhang N, Huang M, Zeng X, et al. CT imaging features of 2019 novel coronavirus (2019-nCoV). Radiology. (2020) 295:202-7. doi: 10.1148/radiol.2020200230

3. Pan F, Ye T, Sun P, Gui S, Liang B, Li L, et al. Time course of lung changes at chest CT during recovery from coronavirus disease 2019 (COVID-19). Radiology. (2020) 295:715-21. doi: 10.1148/radiol.2020 200370

4. Li HW, Zhuo LH, Yan GW, Wang JS, Huang GP, Li JB, et al. High resolution computed tomography for the diagnosis of 2019 novel coronavirus (2019$\mathrm{nCoV}$ ) pneumonia: a study from multiple medical centers in western China. Ann Transl Med. (2020) 8:1158. doi: 10.21037/atm-20-5731

5. Salehi S, Abedi A, Balakrishnan S, Gholamrezanezhad A. Coronavirus disease 2019 (COVID-19): a systematic review of imaging findings in 919 patients. AJR Am J Roentgenol. (2020) 215:87-93. doi: 10.2214/AJR.20. 23034

6. Zhou Z, Guo D, Li C, Fang Z, Chen L, Yang R, et al. Coronavirus disease 2019: initial chest CT findings. Eur Radiol. (2020) 30:4398406. doi: 10.1007/s00330-020-06816-7

7. Li K, Fang Y, Li W, Pan C, Qin P, Zhong Y, et al. CT image visual quantitative evaluation and clinical classification of coronavirus disease (COVID-19). Eur Radiol. (2020) 30:4407-16. doi: 10.1007/s00330-020-06817-6

8. Mondoñedo JR, Sato S, Oguma T, Muro S, Sonnenberg AH, Zeldich $\mathrm{D}$, et al. CT imaging-based low-attenuation super clusters in three dimensions and the progression of emphysema. Chest. (2019) 155:7987. doi: 10.1016/j.chest.2018.09.014

9. Colombi D, Bodini FC, Petrini M, Maffi G, Morelli N, Milanese G, et al. Wellaerated lung on admitting chest CT to predict adverse outcome in COVID-19 pneumonia. Radiology. (2020) 296:E86-96. doi: 10.1148/radiol.2020201433

10. Mo X, Jian W, Su Z, Chen M, Peng H, Peng P, et al. Abnormal pulmonary function in COVID-19 patients at time of hospital discharge. Eur Respir J. (2020) 55:2001217. doi: 10.1183/13993003.01217-2020

11. Xie L, Liu Y, Fan B, Xiao Y, Tian Q, Chen L, et al. Dynamic changes of serum SARS-coronavirus IgG, pulmonary function and radiography in patients recovering from SARS after hospital discharge. Respir Res. (2005) 6:5. doi: 10.1186/1465-9921-6-5

12. Xie L, Liu Y, Xiao Y, Tian Q, Fan B, Zhao H, et al. Follow-up study on pulmonary function and lung radiographic changes in rehabilitating severe acute respiratory syndrome patients after discharge. Chest. (2005) 127:211924. doi: 10.1378/chest.127.6.2119

13. Hui DS, Joynt GM, Wong KT, Gomersall CD, Li TS, Antonio G, et al. Impact of severe acute respiratory syndrome (SARS) on pulmonary function,
81571664, 81871323, and 81801665); the National Natural Science Foundation of Guangdong Province (Grant Number: 2018B030311024); the Scientific Research General Project of Guangzhou Science Technology and Innovation Commission (Grant Number: 201707010328); and the China Postdoctoral Science Foundation (Grant Number: 2016M600145).

\section{ACKNOWLEDGMENTS}

Thanks to Fujifilm (China) Investment Co. Ltd. MIBD team for providing Synapse 3D technical support. Thanks to all the medical workers for their fight against COVID-19 and to the people of the country and the world for their contributions to this campaign. functional capacity and quality of life in a cohort of survivors. Thorax. (2005) 60:401-9. doi: 10.1136/thx.2004.030205

14. Hui DS, Wong KT, Ko FW, Tam LS, Chan DP, Woo J, et al. The 1-year impact of severe acute respiratory syndrome on pulmonary function, exercise capacity, and quality of life in a cohort of survivors. Chest. (2005) 128:224761. doi: 10.1378/chest.128.4.2247

15. Guidelines for the Diagnosis and Treatment of Novel Coronavirus (2019- $n$ CoV) Infection (trial version 7) (in Chinese). National Health Commission of the People's Republic of China (2020)

16. Iwano S, Kitano M, Matsuo K, Kawakami K, Koike W, Kishimoto M, et al. Pulmonary lobar volumetry using novel volumetric computer-aided diagnosis and computed tomography. Interact Cardiovasc Thorac Surg. (2013) 17:5965 doi: 10.1093/icvts/ivt122

17. Cheng Z, Qin L, Cao Q, Dai J, Pan A, Yang W, et al. Quantitative computed tomography of the coronavirus disease 2019 (COVID-19) pneumonia. Radiol Infect Dis. (2020) 7:55-61. doi: 10.1016/j.jrid.2020.04.004

18. Zhou F, Yu T, Du R, Fan G, Liu Y, Liu Z, et al. Clinical course and risk factors for mortality of adult inpatients with COVID-19 in Wuhan, China: a retrospective cohort study. Lancet. (2020) 395:105462. doi: 10.1016/S0140-6736(20)30566-3

19. Xu Z, Shi L, Wang Y, Zhang J, Huang L, Zhang C, et al. Pathological findings of COVID-19 associated with acute respiratory distress syndrome. Lancet Respir Med. (2020) 8:420-2. doi: 10.1016/S2213-2600(20)30076-X

20. Ojha V, Mani A, Pandey NN, Sharma S, Kumar S. CT in coronavirus disease 2019 (COVID-19): a systematic review of chest CT findings in 4410 adult patients. Eur Radiol. (2020) 30:6129-38. doi: 10.1007/s00330-02006975-7

21. Vancheri SG, Savietto G, Ballati F, Maggi A, Canino C, Bortolotto C, et al. Radiographic findings in 240 patients with COVID-19 pneumonia: time-dependence after the onset of symptoms. Eur Radiol. (2020). doi: 10.21203/rs.3.rs-22623/v1

22. Zhang B, Zhang J, Chen H, Chen L, Chen Q, Li M, et al. Novel coronavirus disease 2019 (COVID-19): relationship between chest CT scores and laboratory parameters. Eur J Nucl Med Mol Imaging. (2020) 47:20839. doi: 10.1007/s00259-020-04854-3

23. Ran Y, Xiang L, Huan L, Yanling Z, Xianxiang Z, Qiuxia X, et al. Chest CT severity score: an imaging tool for assessing severe COVID19. Radiol Cardiothorac Imaging. (2020) 2:e200047. doi: 10.1148/ryct.2020 200047

24. Liu J, Chen T, Yang H, Cai Y, Yu Q, hen J, et al. Clinical and radiological changes of hospitalised patients with COVID-19 pneumonia from disease onset to acute exacerbation: a multicentre paired cohort study. Eur Radiol. (2020) 30:5702-8. doi: 10.1007/s00330-020-06916-4

25. Mahdjoub E, Mohammad W, Lefevre T, Debray MP, Khalil A, Study Group. Admission chest CT score predicts 5-day outcome in patients with COVID-19. Intensive Care Med. (2020) 46:1648-50. doi: 10.1007/s00134-02006118-y 
26. Kitano M, Iwano S, Hashimoto N, Matsuo K, Hasegawa Y, Naganawa S. Lobar analysis of collapsibility indices to assess functional lung volumes in COPD patients. Int J Chron Obstruct Pulmon Dis. (2014) 9:134756. doi: 10.2147/COPD.S72616

27. Robbie H, Wells AU, Jacob J, Walsh SLF, Nair A, Srikanthan A, et al. Visual and automated CT measurements of lung volume loss in idiopathic pulmonary fibrosis. AJR Am J Roentgenol. (2019) 213:318-24. doi: 10.2214/AJR.18.20884

28. Ungprasert P, Wilton KM, Ernste FC, Kalra S, Crowson CS, Rajagopalan S, et al. Novel assessment of interstitial lung disease using the "computer-aided lung informatics for pathology evaluation and rating” (CALIPER) software system in idiopathic inflammatory myopathies. Lung. (2017) 195:54552. doi: 10.1007/s00408-017-0035-0

29. Zha L, Shen Y, Pan L, Han M, Yang G, Teng X, et al. Follow-up study on pulmonary function and radiological changes in critically ill patients with COVID-19. J Infect. (2021) 82:159-98. doi: 10.1016/j.jinf.2020 05.040

Conflict of Interest: The authors declare that the research was conducted in the absence of any commercial or financial relationships that could be construed as a potential conflict of interest.

Copyright (c) 2021 Chen, Chen, Liu, Chen, Li, Chen, You, Zhang and Zhang. This is an open-access article distributed under the terms of the Creative Commons Attribution License (CC BY). The use, distribution or reproduction in other forums is permitted, provided the original author(s) and the copyright owner(s) are credited and that the original publication in this journal is cited, in accordance with accepted academic practice. No use, distribution or reproduction is permitted which does not comply with these terms. 